\title{
The Relationship Between Alcohol Problems and Health Functioning of Older Adults in Primary Care Settings
}

\author{
Frederic C. Blow, PhD, ${ }^{* \dagger}$ Maureen A. Walton, $\mathrm{MPH}, \mathrm{PhD},{ }^{\dagger}$ Kristen Lawton Barry, PhD, ${ }^{\dagger}$ \\ James C. Coyne, PhD, Sharon A. Mudd, RN, MS, ${ }^{\dagger}$ and Laurel A. Copeland, MPH*
}

OBJECTIVE: The purpose of this study was to determine the relationship between alcohol use and health functioning in a sample of older adults screened in primary care settings.

DESIGN: A cross-sectional study.

SETTING: Thirty-seven primary care clinics.

PARTICIPANTS: Older adults ( $\mathrm{n}=8578$; aged 55-97) with regularly scheduled appointments in primary care clinics were screened.

MEASUREMENTS: Participants were categorized based on alcohol consumption levels as abstainers, low-risk drinkers, and at-risk drinkers (women: 9 or more drinks/week; men: 12 or more drinks/week). Dependent variables were eight SF-36 health functioning scales.

RESULTS: Sixty-one percent of participants were abstainers, $31 \%$ were low-risk drinkers, and $7 \%$ were at-risk drinkers. ANCOVAs found significant effects of drinking status on General Health, Physical Functioning, Physical Role Functioning, Bodily Pain, Vitality, Mental Health, Emotional Role, and Social Functioning, controlling for age and gender, with low-risk drinkers scoring significantly better than abstainers. At-risk drinkers had significantly poorer mental health functioning than low-risk drinkers. Few significant gender differences were found on SF-36 scales.

CONCLUSIONS: Older adults who are at-risk drinkers may not present with poor physical health functioning. Future studies are needed to determine the relationship between drinking limits for older adults and other areas of physical and psychosocial health. J Am Geriatr Soc 48:769-774, 2000.

Key words: screening, alcohol problems, risky drinking, health promotion, eldexly

This research was supported by NIAAA Grant No. P50-AA07378. Address correspondence and reprint requests to Frederic $\mathrm{C}$. Blow, $\mathrm{PhD}$, University of Michigan, 400 E. Eisenhower Pkwy, Bldg. 2, Suite A, Ann Arbor, MI 48108-3318.

From the "Department of Veterans Affairs Serious Mental Illness Treatment Research and Evaluation Center (SMITREC), Health Services Research and Development (HSR\&D), Ann Arbor, Michigan; †University of Michigan, Department of Psychiatry, Ann Arbor, Michigan; and $¥$ University of Pennsylvania, Departments of Family Medicine and Psychiatry, Philadelphia, Pennsylvania.
Tealth promotion and primary disease prevention among 1 older adults have received growing attention as record numbers of older adults are seeking costly health care for acute and chronic conditions. ${ }^{1,2}$ Targeted early identification and secondary prevention programs for older people have been developed and have been shown to be effective in areas as diverse as cancer, heart disease, smoking, and diet. ${ }^{3-8}$ Potential alcohol problems among older at-risk drinkers have been largely ignored in these efforts with the exception of two brief intervention trials, one recently completed study ${ }^{9}$ and one ongoing study. ${ }^{10}$ Few studies reporting alcohol use among primary care populations have had adequate samples of older adults, especially women. ${ }^{11}$

Alcohol use among older adults is important because what might be considered light or moderate drinking for individuals in their thirties may have untoward health effects in an older person. ${ }^{10,12}$ Comparable amounts of alcohol produce higher blood alcohol levels in older adults than in younger persons and may exacerbate other health problems such as chronic illness, poor nutrition, and polypharmacy. ${ }^{13,14}$ Symptoms of harmful drinking may be less visible among older adults because they may be masked by social, medical, or psychological conditions. Older adults are more likely than younger adults to seek services from their primary care provider; therefore, clinicians working with older patients need to assess alcohol use levels and be aware of the potential health implications of patients' alcohol use. ${ }^{15}$

The relationship between alcohol use levels and health consequences is complicated by varying recommendations for alcohol use cut-off levels and varying definitions of at-risk drinking among older adults. In a large primary care study of patients more than age 60 , Adams, Barry, and Fleming ${ }^{16}$ found that $15 \%$ of the men and $12 \%$ of the women sampled regularly drank in excess of National Institute on Alcohol Abuse and Alcoholism (NIAAA) limits, more than seven drinks/week for women and more than 14 drinks/week for men. Newer NIAAA guidelines now recommend no more than one drink a day, or seven drinks/week for both men and women older than age $65 .{ }^{17}$ These conservative guidelines are consistent with empirical evidence for risk-free drinking among older adults. ${ }^{18}$ However, these NIAAA guidelines do not fully account for findings of decreased risk of coronary artery disease among moderate drinkers. ${ }^{19,20}$ The rationale for setting more conservative guidelines is that vulnerability to alcohol problems is not easily predicted, whereas similar 
decreased risk of coronary artery disease is possible through exercise and diet. ${ }^{8}$ Furthermore, the health costs of untreated alcohol problems may be even greater among older adults who are already at increased risk for many health problems. In contrast to potential protective effects of moderate alcohol consumption, drinking at hazardous levels increases the risk of hypertension ${ }^{21,22}$ and may increase the risk of breast cancer $^{23,24}$ and diabetes, ${ }^{25}$ in this population. Other risks from drinking at hazardous levels include greater risk for harmful drug interactions, injury, depression, memory problems, liver disease, cardiovascular disease, cognitive changes, and sleep problems. ${ }^{26,27}$ Note that definitions of "hazardous" drinking vary by study.

The prevalence of alcohol use and the potential for alcohol-related health problems and decreased health functioning in older primary care populations is a relevant concern of clinicians who work with older adults. The overall interest in health assessment has resulted in the development of several measurement tools, including the Medical Outcome Study (MOS) Short Form Health Survey (SF-36), ${ }^{28}$ to assess perceptions of physical and mental health functioning. The SF-36 has been validated with various age groups, including older adults. ${ }^{29-33}$ None of the published research projects using the SF-36 have studied the relationship between alcohol use patterns and health status in older adulthood. Now, with the availability of validated measures of both health functioning and alcohol use in older adults, the relationship between drinking and health functioning can be determined. The purpose of this study was to examine the relationship between alcohol use and health functioning in a sample of older adults screened in primary care settings.

\section{METHODS}

Study procedures were approved and conducted in compliance with the University of Michigan's Institutional Review Board for Human Subjects guidelines. Older patients in 37 primary care clinics in southeastern Michigan and northwest Ohio (Toledo area) were asked to complete a health survey, administered by a trained research assistant, while they waited for their primary care physician appointments. Rolling site recruitment took place over a period of over 3 years. Patients were sampled from the time of each site's enrollment until the yield of new older adult patients was exhausted. Participants who agreed to complete the health screening survey were told they might be called for future studies; however, efforts were made to reduce sensitization of respondents to issues of alcohol use by embedding alcohol questions among health-related questions such as smoking, nutrition, and exercise. The survey required about $15 \mathrm{~min}-$ utes to complete. Participants were given the option of completing the paper-and-pencil questionnaire themselves or having the trained research assistant administer it to them. Patients identified as at-risk drinkers were invited to participate in a clinical trial of an elder-specific brief intervention. This paper reports data from the screening phase of the project. The clinical trial portion of the study is in progress.

Primary care clinics in this study included single physician offices, multi-physician HMO clinics, and three outpatient general medicine clinics at Veterans Affairs (VA) medical centers (providing $48 \%$ of the sample). Clinics were sampled both concurrently, with up to 20 research assistants surveying patients on a given day, and sequentially over the 3-year period. Research assistants were stationed at clinics one or more days a week, depending on patient flow. Older adults with regularly scheduled appointments were approached by research assistants and asked to participate. To respect the possible sensitivity of patients with literacy or physical limitations, patients were asked if they would like to complete the questionnaire themselves or have assistance. The majority of the surveys were self-administered $(85 \%)$ in the waiting rooms. The remaining $15 \%$ of the sample were interviewed by a research assistant, using response cards whenever necessary, in order to protect confidentiality. Assisted interviews were conducted at the participant's request, typically because of visual difficulties, illiteracy, or physical disabilities that precluded writing.

Refusal reasons were compiled in a log by the research assistants as they occurred. Reasons for refusal included: unknown/would not give reason $(49 \%)$, too ill $(18 \%)$, hostile toward research $(11 \%)$, and would take too much time $(9 \%)$. In addition, miscellaneous reasons for refusal included expecting monetary compensation for completion and willingness to complete at another visit $(13 \%)$. It was not possible to calculate an accurate refusal rate for several reasons. First, patients often refused on one occasion but said they would participate at their next visit. A participant could be counted twice, as a participant and a refusal. Second, it was not possible to determine accurately if a person refused on multiple occasions because they were approached by different research assistants. It was not possible to determine if each refusal recorded represented a unique individual. Third, because research assistants were not able to ascertain the age of patients without potentially offending them, anyone appearing middle-aged or older was approached. Surveys of participants less than age 55 were excluded from the study $(n=$ 3668). Thus, refusal could also include participants ineligible based on age. A total of 5174 refusals were recorded during the 3 years of recruitment.

\section{Participant Demographics}

Between February 1995 and February 1998, a total of 8578 older adults were screened in primary care settings. Forty-three percent $(\mathrm{n}=3676)$ of those screened were women. The mean age of participants was $68.8(\mathrm{SD}=7.5$ ), with a range from 55 to 97 years. Seventy-seven percent $(77.4 \%)$ were white, $19.4 \%$ were black, and only $3.2 \%$ identified themselves as Hispanic, Asian, Native American, or other. Sixty-four percent $(64.6 \%)$ were married or cohabiting, $18.0 \%$ were widowed, $13.7 \%$ divorced/separated, and $3.7 \%$ never married. Forty percent $(40.5 \%)$ had completed high school, $26.5 \%$ completed less than high school, and $33.0 \%$ had completed at least some college.

\section{Measures}

The health survey for the study included the eight scales from the Medical Outcomes Study Short Form-36 (MOS SF-36), demographics, and health habits questions, including alcohol use in the past 3 months.

The MOS SF-36 is a 36-Item General Health Survey that was originally developed for use with adults as a 20 -item scale for the Medical Outcome Study from more detailed measures utilized in the Rand Health Experiment. ${ }^{34,35}$ It was subsequently expanded to 36 items. As currently defined, the SF-36 measures eight domains: the General Health Index, Physical Functioning, Physical Role Functioning, Bodily Pain, Vitality, Mental Health, Emotional Role Functioning, 
and Social Functioning (see Appendix A for items). The SF-36 has published norms for these various subscales. ${ }^{36}$ Likertscaled items assessed health status. Negatively keyed items were recoded. Within each domain, scores were summed, and the total was then expressed as a percentage of the maximum possible. Higher scores indicated better functioning, and five points "defines differences that are clinically and socially relevant." ${ }^{37}$ The SF-36 subscales were the dependent variables of interest in the current study.

Questions about alcohol use included lifetime use, past year use, and reasons for quitting (if currently abstinent). Three alcohol consumption questions asked in regard to the previous 3-month period covered average frequency, average quantity, and binge frequency. The number of drinking days per week was multiplied by the reported number of drinks per drinking day to form the alcohol use measure (number of standard drinks/week; a standard drink $=12 \mathrm{oz}$. of beer, $1 \frac{1 / 2}{2}$ oz. hard liquor, or $5 \mathrm{oz}$. wine). Cut-off levels for at-risk drinking were at least nine drinks per week for women and at least 12 drinks per week for men. These alcohol use cut-offs were lower than those recommended by Sanchez-Craig et al. z]38 for younger samples because of age-related changes in alcohol sensitivity and tolerance. The alcohol frequencyquantity measure was used to create three drinking level categories: abstainers, low-risk drinkers (up to the genderspecific hazardous drinking levels just mentioned), and atrisk drinkers.

Binge drinking was defined as drinking four or more standard drinks on a drinking occasion. Binge drinking criteria for older adults were set at a lower level than is recommended for younger adults $(5+$ standard drinks/occasion). To meet criteria for binge drinking in this study, a participant had to report drinking four or more drinks at a time at least twice in the past 3 months.

\section{Analyses}

Chi-square analyses and Student's $t$ tests were used to identify demographic differences. A series of analyses of covariance (ANCOVAs) assessed differences in health outcomes by drinking status and gender, with Tukey's adjustment for simultaneous post hoc comparisons. Age was entered as a covariate. In the ANCOVAs, a significant main effect of a factor, such as drinking status, would indicate that when the participants were grouped by their drinking status, group mean scores on the MOS scales were significantly different for at least one linear combination. In the post hoc analyses, exact $P$ values are not generated but are assessed for significance at the alpha $=.05$ level. For the two-group parametric comparisons, $t$ tests were run. A criterion alpha level of .05 was used throughout. Note that the number of participants who completed the SF-36 was smaller than the number who completed the drinking questions because the SF-36 was positioned at the end of the questionnaire, and some patients were unable to complete that section during their clinic visit because of time constraints.

\section{RESULTS}

\section{Prevalence of Alcohol Use}

Of the 7910 participants who answered the questions about alcohol use, $7.4 \%(n=589)$ drank at or above the defined at-risk drinking level of nine or more drinks per week for women and 12 or more drinks per week for men (see Table 1). Respondents were classified according to their drinking as $61.3 \%$ abstainers ( $n=4848), 31.3 \%$ low-risk drinkers $(n=2473), 7.4 \%$ at-risk drinkers $(n=589)$. Binge drinking ranged from 0 to $10+$ times in the last three months. Of those currently reporting any alcohol use, a total of $24.5 \%$ ( $\mathrm{n}=745$ ) met binge drinking criteria.

The average age of respondents differed significantly by drinking group $(P<.05$ with Tukey's adjustment for simultaneous pairwise comparisons). Abstainers were significantly older at $69.24 \pm 7.57$ than moderate drinkers (age 67.82 \pm 7.63 ), and at-risk drinkers were youngest at 67.61 years \pm 6.43 .

Significant gender differences were found, with $10.6 \%$ of the men but only $3.4 \%$ of the women identified as at-risk drinkers (chi-square $=180.69 ; d f=2 ; P<.001$ ). The large size of the abstainer group $(61.3 \%)$ prompted us to examine prior drinking history to determine reasons for abstention. Of the $\mathbf{4 3 0 6}$ abstainers who reported prior drinking status, $\mathbf{7 9 . 9 \%}$ reported ever drinking, leaving 865 lifetime abstainers. Of those who reported reasons for quitting, $43.9 \%$ quit for health reasons unrelated to alcohol; $5.6 \%$ quit for alcohol-related reasons, and $50.5 \%$ quit for other reasons, including cost, taste, and religious and social considerations. These subtypes of abstainers are the subject of another report currently in preparation.

Table 1. Drinking Measures and Alcohol Risk Group by Gender

\begin{tabular}{|c|c|c|c|c|}
\hline Measure & $\begin{array}{c}\text { Men } \\
(n=4869) \\
\text { Mean } \pm S D\end{array}$ & $\begin{array}{c}\text { Women } \\
(n=3676) \\
\text { Mean } \pm \text { SD }\end{array}$ & $\begin{array}{l}\text { Statistic }(d f) \\
t \text { Value }\end{array}$ & $P$ Value \\
\hline $\begin{array}{l}\text { Drinks/week } \\
\text { Binge drinking* }\end{array}$ & $\begin{array}{l}3.90 \pm 10.53 \\
0.81 \pm 2.19\end{array}$ & $\begin{array}{l}1.26 \pm 4.88 \\
0.16 \pm 0.85\end{array}$ & $\begin{array}{l}14.81(6655.1) \\
17.21(6135.9)\end{array}$ & $\begin{array}{l}<.001 \\
<.001\end{array}$ \\
\hline Alcohol Risk Group ${ }^{\dagger}$ & Percentage & Percentage & $\begin{array}{c}\text { Chi-Square } \\
180.69 \text { (2) }\end{array}$ & .001 \\
\hline $\begin{array}{l}\text { Abstainer } \\
\text { Light/moderate drinkers } \\
\text { At-risk drinkers }\end{array}$ & $\begin{array}{l}56.49 \\
32.94 \\
10.57\end{array}$ & $\begin{array}{r}67.49 \\
29.12 \\
3.40\end{array}$ & & \\
\hline
\end{tabular}

"Occasions in the past 3 months.

${ }^{+}$Abstainers $=$past year; Light/moderate drinkers $=0$ to 8 (women) and 0 to 11 (men) drinks per week; At-risk drinkers $=9+($ women) and $12+($ men) drinks per week. 


\section{Alcohol Risk, Gender, and Health Functioning}

In the analysis of covariance analyses, there were statistically significant main effects of drinking group on all eight MOS SF-36 scales assessed: General Health $(P<.001)$, Physical Functioning $(P<.05)$, Physical Role Functioning $(P<.001)$, Bodily Pain $(P<.001)$, Vitality $(P<.001)$, Mental Health $(P<.001)$, Emotional Role Functioning $(P<$ $.02)$, and Social Functioning $(P<.001)$. In all of these measures of health functioning, the low risk drinkers scored significantly better than the abstainers $(P<.05$ post hoc). On all but Mental Health, the at-risk drinkers also scored significantly better than the abstainers $(P<.05$ post hoc). The only scale on which low-risk drinkers scored significantly better than at-risk drinkers was Mental Health $(P<.05$ post hoc). As mentioned earlier, a 5-point difference on the MOS SF-36 scales constitutes a clinically significant difference (see Table 2). Findings for Vitality and Mental Health Functioning did not meet this criterion.

There were statistically significant main effects of gender on General Health $(P<.001)$, Physical Functioning $(P<$ $.03)$, Physical Role Functioning $(P<.01)$, Mental Health $(P<.05)$, and Social Functioning $(P<.01)$. However, using a 5-point difference as the criterion, only on General Health Functioning did these gender differences reach clinical significance, with women having better General Health than men (see Table 3).

\section{DISCUSSION}

Prevalence rates of drinking reported by older adults in this study are consistent with national surveys. ${ }^{10}$ The rates of hazardous or at-risk drinking are slightly less than those found by Sanchez-Craig et al..$^{38}$ and Adams et al. ${ }^{16}$ These differences may be attributable to geographical variation or different screening questionnaire items. Reports of at-risk drinking vary across prevalence studies based on variations in risk drinking definitions in addition to the set of screening questions used, time periods sampled, setting for questionnaire administration, and area of the country or world in the sample.

More than $10 \%$ of the men and $3 \%$ of the women aged 55 and older drank at levels considered at-risk for alcohol problems. For this study, at-risk drinking was defined as nine or more alcoholic drinks per week for women, and 12 or more alcoholic drinks per week for men. The cut-offs used for older adults (age 55 and older) in this study were based on a synthesis of the following research. First, hazardous drinking for younger persons is typically defined as $\mathbf{1 2}$ or more drinks per week for women and 15 or more drinks per week for men. ${ }^{38}$ Second, age-related changes in lean muscle to body fat ratios result in higher blood alcohol levels among older adults ${ }^{13}$ and older adults experience symptoms of alcohol abuse or dependence at lower consumption levels. ${ }^{18}$ More clarification is needed in the literature regarding definitions of at-risk or hazardous drinking based on physical and psychosocial health consequences. ${ }^{39}$

In this sample, older adults who abstained from alcohol in the past year reported the poorest overall physical functioning compared with moderate and at-risk drinkers. This is consistent with findings by Nelson et al. ${ }^{40}$ that older women with health problems were more likely to abstain from alcohol. The likely reason for this is that drinking is contraindicated for some health problems and for many medications prescribed commonly for this age group. Furthermore, older patients experiencing more negative health consequences may discontinue alcohol use on their own.

In contrast, older adults who drank in moderation, consuming less than eight drinks/week (women) or less than 12 drinks/week (men), reported the best health functioning. In all physical health functioning scales from the SF-36, the low-risk drinkers scored significantly better than the abstainers. Although results are consistent with studies showing protective cardiovascular effects from moderate alcohol consumption, recommendations about possible benefits of moderate drinking on physical and mental health cannot be made based on this cross-sectional study. Future longitudinal studies are needed to determine the causal nature of the relationship between alcohol use and health functioning.

Older at-risk drinkers were not distinguished by physical and mental health functioning deficits. Although at-risk drinkers had statistically poorer mental health functioning than low-risk drinkers, the magnitude of this difference was not clinically significant. Thus, results from this study do not offer support for the drinking limits for older adults used in this study (less than 8 drinks/week (women) or less than 12 drinks/week (men)) in terms of deficits in health functioning among a primary care population. However, older adults drinking above these limits may place themselves at risk for a variety of other alcohol-related consequences not measured by this study ${ }^{18}$ Furthermore, the population for this study is likely to be less severe in terms of both drinking and health

Table 2. Means for Health Functioning Scores (from the MOS SF-36) by Alcohol Risk Group

\begin{tabular}{lccccc}
\multicolumn{1}{c}{ Sub-scale } & $\mathrm{n}$ & $\begin{array}{c}\text { Abstainers } \\
\text { Mean } \pm \text { SD }\end{array}$ & $\begin{array}{c}\text { Light Drinkers* } \\
\text { Mean } \pm \text { SD }\end{array}$ & $\begin{array}{c}\text { At-Risk Drinkers } \\
\text { Mean } \pm \text { SD }\end{array}$ & $\begin{array}{c}\text { Main Effect } \\
P \text { Value }\end{array}$ \\
\hline General health functioning & 7394 & $54.38 \pm 22.50$ & $62.39 \pm 20.99$ & $60.07 \pm 21.20$ \\
Physical functioning & 6878 & $60.75 \pm 29.54$ & $71.27 \pm 26.02$ & $68.49 \pm 27.50$ & $<.001$ \\
Physical role functioning & 6445 & $51.11 \pm 42.01$ & $59.93 \pm 40.71$ & $60.79 \pm 39.94$ & $<.001$ \\
Bodily pain & 7478 & $59.22 \pm 28.03$ & $66.54 \pm 26.05$ & $64.81 \pm 26.90$ & $<.001$ \\
Vitality & 7391 & $53.04 \pm 22.99$ & $58.13 \pm 20.75$ & $58.37 \pm 20.85$ & $<.001$ \\
Mental health functioning & 7374 & $73.37 \pm 18.77$ & $76.80 \pm 15.79$ & $74.97 \pm 18.25$ & $<.001$ \\
Emotional role functioning & 6407 & $76.23 \pm 36.43$ & $83.55 \pm 30.45$ & $80.24 \pm 33.67$ & $<.02$ \\
Social health functioning & 6494 & $80.05 \pm 25.12$ & $85.70 \pm 21.36$ & $85.62 \pm 21.73$ & $<.001$ \\
\hline
\end{tabular}

* 0 to 8 (women) and 0 to 11 (men) drinks per week.

t $9+$ (women) and $12+$ (men) drinks/week. 
Table 3. Means and Standard Deviations for Health Functioning Scores (from the MOS SF-36) by Gender

\begin{tabular}{lcccc}
\hline \multicolumn{1}{c}{ Sub-scale } & $\mathrm{n}$ & Women & Men & $\begin{array}{c}\text { Main Effect } \\
P \text { Value }\end{array}$ \\
\hline General health functioning & 7394 & $60.34 \pm 21.64$ & $55.17 \pm 22.44$ & $<.001$ \\
Physical functioning & 6878 & $65.61 \pm 27.84$ & $64.20 \pm 29.33$ & $<.03$ \\
Physical role functioning & 6445 & $57.09 \pm 41.06$ & $53.12 \pm 42.02$ & $<.01$ \\
Bodily pain & 7478 & $61.43 \pm 26.89$ & $62.34 \pm 28.03$ & $>.05$ \\
Vitality & 7391 & $55.20 \pm 21.59$ & $54.97 \pm 22.80$ & $>.05$ \\
Mental health functioning & 7374 & $73.66 \pm 17.40$ & $75.32 \pm 18.23$ & $<.05$ \\
Emotional role functioning & 6407 & $80.21 \pm 33.44$ & $78.09 \pm 35.22$ & $>.05$ \\
Social health functioning & 6494 & $84.03 \pm 22.98$ & $81.09 \pm 24.38$ & $<.01$ \\
\hline
\end{tabular}

functioning; studies examining older adult samples from other settings may find very different results.

Several statistically significant gender effects were found, but only in the case of general health functioning did these differences appear clinically relevant, given their magnitude. Although gender differences in health functioning were not profound in this primary care sample, in all cases, women scored better than men. This finding is consistent with the literature where, even among older persons with drinking problems, women, with their better connections to family and friends, seem to be healthier than men. ${ }^{41,42}$

The strengths of this study include the large sample size, the use of diverse primary care settings for data collection, and the measurement of health functioning in relation to alcohol use. Limitations include omitting patients who were too ill, infirm, or cognitively impaired to complete questionnaires or interviews. Unfortunately, there is little information about the nonparticipants. Therefore, the reported use of alcohol may be an underestimate; however, self-report data on usual alcohol use are generally considered reliable, ${ }^{43,44}$ and embedding questions about alcohol use in a questionnaire about health habits is often done in primary care settings. ${ }^{16}$ There are more costly and potentially more accurate methods to elicit drinking data, such as the Time-Line-FollowBack (TLFB) diary method, but these approaches are too resource- and time-intensive for primary care settings. The use of brief, efficient, self-report methods to obtain alcohol consumption data in this venue is well established. ${ }^{43,44}$

In summary, low-risk drinkers had the best health functioning and abstainers had the poorest health functioning. Whether this finding supports the benefits of moderate alcohol consumption can not be determined by this study. Furthermore, low-risk and at-risk drinkers were not generally distinguished by differences in health functioning. The appropriateness of the drinking limits used in this study, in terms of understanding health functioning among a primary care sample, is not clear. Older adults at risk for alcohol problems may not present in primary care with poor physical health functioning. Given the prevalence of at-risk drinking among older adults in primary care settings and the potential impact of risk drinking on psychosocial areas not assessed in this study, brief targeted screening and interventions for alcohol could be of benefit to older adults receiving health care in primary care settings. Primary care screening and intervention can be a cost-effective method to improve the quality of life for this growing, resource-intensive patient population. Further studies with larger minority representation should be directed toward the exploration of factors that influence physical health and the role of alcohol use in racial subgroups. There is still much to learn about the drinking patterns of older persons and trajectories into later life as well as the relationship between drinking limits and health functioning in both primary care and substance abuse treatment populations.

\section{APPENDIX A}

\section{ITEMS IN THE MEDICAL OUTCOMES STUDY SF-36 SUBSCALES}

\section{General Health Index}

In general, would you say your health is: excellent/very good/good/fair/poor; I seem to get sick a little easier than other people; I am as healthy as anybody I know; I expect my health to get worse; My health is excellent.

\section{Physical Functioning}

Does your health limit you in these activities? If so, how much? Vigorous activities, such as running, lifting heavy objects, participating in strenuous sports; Moderate activities, such as moving a table, bowling or playing golf; Lifting or carrying groceries; Climbing several flights of stairs; Climbing one flight of stairs; Bending, kneeling, or stooping; Walking more than a mile; Walking several blocks; Walking one block; Bathing and dressing.

\section{Physical Role Functioning}

During the past 4 weeks, have you had any of the following problems with your work or other regular daily activities as a result of your physical health? Cut down on the amount of time you spent on work or other activities; Accomplished less than you would like; Were limited in the kind of work or other activities; Had difficulty performing the work or other activities (for example, it took extra effort).

\section{Bodily Pain}

How much bodily pain have you had during the past 4 weeks? During the past 4 weeks, how much did pain interfere with your normal work including both work outside the home and housework?

\section{Vitality}

How much of the time during the past 4 weeks. . . did you feel full of pep? Did you have a lot of energy? Did you feel worn out? Did you feel tired? 


\section{Mental Health}

How much of the time during the past 4 weeks have you. . . been a very nervous person? Have you felt so down in the dumps that nothing could cheer you up? Have you felt calm and peaceful? Have you felt downhearted and blue? Have you been a happy person?

\section{Emotional Role Functioning}

During the past 4 weeks, have you had any of the following problems with your work or other regular daily activities as a result of any emotional problems (such as feeling depressed or anxious)? Cut down the amount of time you spent on work or other activities; Accomplished less than you would like; Didn't do work or other activities as carefully as usual?

\section{Social Functioning}

During the past 4 weeks, to what extent have your physical health or emotional problems interfered with your normal social activities with family, friends, neighbors, or groups? During the past 4 weeks, how much of the time have your physical health or emotional problems interfered with your social activities (like visiting with friends, relatives, etc.)?

\section{REFERENCES}

1. Lusky RA. Anticipating the needs of the U.S. aged in the 21 st century: Dilemmas in epidemiology, gerontology, and public policy. Soc Sci Med 1986; 23:1217-1227.

2. Waldo DR, Sonnefeld ST, McKusick DR, Arnett RH III. Health expenditures by age group, 1977 and 1987. Health Care Financ Rev 1989;10:111-120.

3. Coambs RB, Li S, Kozlowski LT. Age interacts with heaviness of smoking in predicting success in cessation of smoking. Am J Epidemiol 1992;135:240-246.

4. LaCroix AZ, Omenn GS. Older adults and smoking. Clin Geriatr Med 1992; 8:69-87.

5. Blumenthal JA, Levenson RM. Behavioral approaches to secondary prevention of coronary heart disease. Circulation 1987;76:1130-137.

6. Davenport J, Whittaker $\mathrm{K}$. Secondary prevention in elderly survivors of heart attacks. Am Fam Physician 1988;38:216-224.

7. Levine $\mathrm{R}$, Tenner $\mathrm{S}$, Fromm $\mathrm{H}$. Prevention and early detection of colorectal cancer (see comments). Am Fam Physician 1992;45:663-668.

8. Gambrell RD Jr. Cancer in the older woman: Diagnosis and prevention. Geriatrics $1988 ; 43: 27-32: 35-36$.

9. Fleming MF, Manwell LB, Barry KL et al. Guiding older adult lifestyles (Project GOAL): The effectiveness of brief physician advice for alcohol problems in older adults. J Fam Pract, in press.

10. Blow FC. The spectrum of alcohol interventions for older adults. In: Alcohol Problems and Aging. NIAAA Research Monograph, 1998, 33, pp 373-396. (DHHS Pub. No. 98-4163).

11. Fleming MF, Barry KL, Manwell LB et al. Brief physician advice for problem alcohol drinkers. A randomized controlled trial in community-based primary care practices. JAMA 1997;277:1039-1045.

12. Blow FC. Alcohol. In: Substance Abuse Among Older Adults. Treatment Improvement Protocol (TIP) Series, 1998, 26, pp 13-28. (DHHS Pub. No. (SMA 98-3179).

13. Vogel-Sprott M, Barrett P. Age, drinking habits, and the effects of alcohol. J Stud Alcohol 1984;45:517-521.

14. Vestal RE, McGuire EA, Tobin JD et al. Aging and ethanol metabolism. Clin Pharmacol Ther 1977;21:343-354.

15. Blow FC. Identification, screening, and assessment. In: Substance Abuse Among Older Adults. Treatment Improvement Protocol (TIP) Series 1998, 26, pp 47-64. (DHHS Pub. No. (SMA):98-3179).

16. Adams W, Barry KL, Fleming MF. Screening for alcohol use in older primary care patients. JAMA 1996;279:1964-1967.

17. National Institute of Alcoholism and Alcohol Abuse (NIAAA). The Physicians' Guide to Helping Patients with Alcohol Problems. NIH Publ. No. 1995:95-3769.
18. Chermack ST, Blow FC, Hill EM, Mudd SA. The relationship between alcohol symptoms and consumption among older drinkers. Alcohol Clin Exp Res 1996;20:1153-1158.

19. Ashley MF, Ferrence R, Room R et al. Moderate drinking and health. Implications of recent evidence. Can Fam Phys 1997;43:687-694.

20. Makela P, Valkonen T, Poikolainen K. Estimated numbers of deaths from coronary heart disease "caused" and "prevented" by alcohol: An example from Finland. J Stud Alcohol 1997;58:455-463.

21. MacMahon S. Alcohol consumption and hypertension. Hypertension 1987 ; 9:111-121.

22. Klatsky AL, Friedman CD, Armstrong MA. The relationship between alcoholic beverage use and other traits to blood pressure: A new Kaiser Permanente study. Circulation 1986;73:628-636.

23. Schatzkin A, Longnecker MP. Alcohol and breast cancer. Cancer 1994;74: 1101-1110.

24. Longnecker MP. Alcoholic beverage consumption in relation to risk of breast cancer: Meta-analysis and review. Cancer Causes Control 1994;5:73-82.

25. Holbrook TL, Barrett-Connor E, Wingard DL. A prospective populationbased study of alcohol use and non-insulin-dependent diabetes mellitus. Am J Epidemiol 1990;132:902-909.

26. Gambert SR, Katsoyannis KK. Alcohol-related medical disorders of older heavy drinkers. In: Beresford T, Gomberg ESL, eds. Alcohol and Aging. New York: Oxford University Press, 1995, pp 70-81.

27. Liberto JG, Oslin DW, Ruskin PE. Alcoholism in older persons: A review of the literature. Hosp Commun Psychiatry 1992;43:975-984.

28. Ware JE Jr, Sherbourne CD. The MOS 36-Item Short-Form Health Survey (SF-36): I. Conceptual framework and item selection. Med Care 1992;30: 473-481.

29. McHorney CA. Measuring and monitoring general health status in elderly persons: Practical and methodological issues in using the SF-36 health survey. Gerontologist 1996;36:571-583.

30. Booth BM, Blow FC, Cook CAL. Functional impairment and co-occurring psychiatric disorders in medically hospitalized men. Arch Intern Med 1998; 158:1551-1559.

31. Beusterien KM, Steinwald B, Ware JE Jr. Usefulness of the SF-36 health survey in measuring health ourcomes in the depressed elderly. J Geriatr Psychiatry Neurol 1996;9:13-21.

32. Andresen EM, Patrick DL, Carter WB, Malmgren JA. Comparing the performance of health status measures for healthy older adults. J Am Geriatr Soc 1995;43:1030-1034.

33. Lyons RA, Perry HM, Littlepage BNC. Evidence for the validity of the shortform 36 questionnaire (SF-36) in an elderly population. Age Ageing 1994; 23:182-184.

34. Tarlov AR, Ware JE Jr, Greenfield S et al. The Medical Outcomes Study: An application of methods for monitoring the results of medical care. JAMA 1989;262:925-930.

35. Stewart AL, Hays RD, Ware JE Jr. The MOS Short-Form General Health Survey. Reliability and validity in a patient population. Med Care 1988;26: 724-735.

36. McHorney CA, Ware JE, Raczek AE The MOS 36-Item Short-Form Health Survey (SF-36): II. Psychometric and clinical tests of validity of measuring physical and mental health constructs. Med Care 1993;31:247-263.

37. Ware JE, Snow KK, Kosinski M, Gandek B. SF-36 Health Survey Manual and Interpretation Guide. Boston, MA: The Health Institute, New England Medical Center, 1993, 7:12.

38. Sanchez-Craig M, Wilkinson DA, Davila R. Empirically based guidelines for moderate drinking: 1-year results from three studies with problem drinkers. Am J Public Health 1995;85:823-828.

39. Walton MA, Mudd SA, Blow FC et al. Stability in the drinking habits of older problem drinkers recruited from the community. J Subst Abuse Treat, 2000;18:169-177.

40. Nelson HD, Nevitt MC, Scott JC et al. Smoking, alcohol, and neuromuscular and physical function of older women. JAMA 1994;272:1825-1831.

41. Gomberg ESL. Drugs, alcohol and aging. In: Kozlowski LT, Annis HM et al., eds. Research Advances in Alcohol and Drug Problems. New York: Plenum Press, 1990 pp 171-213.

42. Brennan PL, Moos RH, Kim JY. Gender differences in the individual characteristics and life contexts of late-middle-aged and older problem drinkers. Addiction 1993;88:781-790.

43. Midanik $L$. The validity of self-reported alcohol consumption and alcohol problems: A literature review. Br J Addict 1982;77:357-382.

44. Sobell LC, Sobell MB. Self-report issues in alcohol abuse: State of the art and future directions. Behav Assess 1990;12:91-106. 